\title{
Spatial and spectral mode mapping of a dielectric nanodot by broadband interferometric homodyne scanning near-field spectroscopy
}

\author{
Jinxin Zhan, ${ }^{a}$ Wei Wang, ${ }^{b}$ Jens Brauer, ${ }^{a}$ Lukas Schmidt-Mende, ${ }^{b}$ Christoph Lienau, ${ }^{a, \star}$ and Petra Gro $\beta^{a, \star}$ \\ ${ }^{a}$ Carl von Ossietzky Universität, Institut für Physik and Center of Interface Science, Oldenburg, Germany \\ 'University of Konstanz, Department of Physics, Konstanz, Germany
}

\begin{abstract}
We investigate the optical properties of nanostructures of antimony sulfide $\left(\mathrm{Sb}_{2} \mathrm{~S}_{3}\right)$, a direct-bandgap semiconductor material that has recently sparked considerable interest as a thin film solar cell absorber. Fabrication from a nanoparticle ink solution and two- and three-dimensional nanostructuring with pattern sizes down to $50 \mathrm{~nm}$ have recently been demonstrated. Insight into the yet unknown nanoscopic optical properties of these nanostructures is highly desired for their future applications in nanophotonics. We implement a spectrally broadband scattering-type near-field optical spectroscopy technique to study individual $\mathrm{Sb}_{2} \mathrm{~S}_{3}$ nanodots with a 20-nm spatial resolution, covering the range from 700 to $900 \mathrm{~nm}$. We show that in this below-bandgap range, the $\mathrm{Sb}_{2} \mathrm{~S}_{3}$ nanostructures act as high-refractive-index, low-loss waveguides with mode profiles close to those of idealized cylindrical waveguides, despite a considerable structural disorder. In combination with their high above-bandgap absorption, this makes them promising candidates for applications as dielectric metamaterials, specifically for ultrafast photoswitching.
\end{abstract}

Keywords: near-field spectroscopy; scattering-type near-field optical microscopy; tip-modulation SNOM; dielectric nanostructure; thin film patterning.

Received Apr. 2, 2020; revised manuscript received Aug. 4, 2020; accepted for publication Aug. 5, 2020; published online Aug. 25, 2020.

(c) The Authors. Published by SPIE and CLP under a Creative Commons Attribution 4.0 Unported License. Distribution or reproduction of this work in whole or in part requires full attribution of the original publication, including its DOI.

[DOI: $10.1117 / 1 . A P \cdot 2 \cdot 4.046004]$

\section{Introduction}

Stibnite (antimony sulfide, $\mathrm{Sb}_{2} \mathrm{~S}_{3}$ ) is currently gaining increasing attention as an emerging photovoltaic material. ${ }^{1,2}$ Considerable efforts are being undertaken to advance the fabrication and structural characterization ${ }^{2-5}$ of these materials in attempts to improve their photovoltaic performance.$^{6-8}$ Annealing at moderate temperatures of typically above $250^{\circ} \mathrm{C}$ activates crystallization of the initially amorphous material and decreases the bandgap energy from 2.24 to $1.73 \mathrm{eV}$ while maintaining a high abovebandgap absorption coefficient of $>5 \times 10^{4} \mathrm{~cm}^{-1}$. ${ }^{2}$ This makes the semiconductor $\mathrm{Sb}_{2} \mathrm{~S}_{3}$ a material which not only can be synthesized from earth-abundant and nontoxic constituents but is strongly absorbing across the entire visible spectrum. The combination of a direct bandgap and a high absorption coefficient

*Address all correspondence to Christoph Lienau, E-mail: christoph.lienau @ uol.de; Petra Groß, E-mail: petra.gross@uol.de leads to considerable photoconductivity, enabling applications in optoelectronics. ${ }^{9}$ Power conversion efficiencies of up to $7.5 \%$ have been demonstrated for an $\mathrm{Sb}_{2} \mathrm{~S}_{3}$-sensitized heterojunction solar cell.

For future applications, it is particularly beneficial that comparatively simple and cheap ink-based methods can be used to create $\mathrm{Sb}_{2} \mathrm{~S}_{3}$ nanoparticles in large-area thin films. ${ }^{10,11}$ As recently demonstrated, nanopatterning via direct electron beam lithography (EBL) writing enables the creation of 2D and 3D nanometric structures of various shapes. ${ }^{9}$ The availability of easy structuring, together with the possibility of changing the refractive index on an ultrafast time scale by injecting free carriers, makes the material an interesting candidate for the realization of photoswitchable metasurfaces. ${ }^{12}$ For this, a high optical quality of $\mathrm{Sb}_{2} \mathrm{~S}_{3}$ nanostructures is an important prerequisite that has not yet been investigated. Since the structural characterization of the bulk material shows polycrystalline grains with typical sizes of tens of $\mathrm{nm}$, and the optical spectra show 
a sizeable sub-bandgap absorption, this may indicate a high crystal defect density. ${ }^{2}$ As such, a nanoscale optical characterization of individual nanoparticles seems particularly desirable.

For these reasons, we report here a first experimental study of the optical properties of $\mathrm{Sb}_{2} \mathrm{~S}_{3}$ nanostructures. Specifically, we aim at probing sub-bandgap waveguiding in nanodots fabricated with EBL to explore their potential as dielectric metamaterials. For this, we spatially image the optical modes of such nanoparticles using a novel, spectrally broadband scattering-type scanning near-field optical microscopy (sSNOM) $)^{13-15}$ technique. Raster-scanning a sharp scattering or collecting tip that is brought into the sample's near field has in the past been applied to mapping the evanescent field distribution of a multitude of different samples, such as waveguides and microresonators, ${ }^{16,17}$ photonic crystal microcavities, ${ }^{18}$ single plasmonic nanoparticles, ${ }^{19-23}$ and, more recently, all-dielectric nanostructures. ${ }^{24,25}$ Ideally, the recorded intensity distribution provides a map of the local optical density of states (LDOS), ${ }^{21,26-31}$ with a spatial resolution of $20 \mathrm{~nm}$ or below. ${ }^{14,32-34}$ The expansion of sSNOM to broad-bandwidth spectroscopy in the visible spectral range, however, is challenging. Unless advanced, virtually background-free techniques such as plasmonic nanofocusing ${ }^{31,35-37}$ are used, this requires efficient background suppression based on tip-sample distance modulation and near-field enhancement using homodyne or heterodyne mixing. ${ }^{38-43}$

Here, we solve these challenges by detecting tip-modulated near-field spectra after homodyne-mixing with a reference field using a monochromator followed by a fast CCD line camera. The camera line readout rate of $210 \mathrm{kHz}$ is sufficiently fast to enable extraction of complete spectra at up to the fourth harmonic of the tip-sample modulation frequency. We obtain background-free near-field spectra in the spectral range from 720 to $900 \mathrm{~nm}$. The SNOM maps recorded across the $\mathrm{Sb}_{2} \mathrm{~S}_{3}$ nanoparticles reveal that they act as high-refractive index, dielectric waveguides, despite their irregular surface apparent in structural studies. They support waveguide-like modes with the electric field strongly confined in the nanodots and mode profiles matching those of idealized cylindrical waveguides, which makes them promising structures for applications in nanophotonics and specifically creates interest in future studies of their nonlinear properties.

\section{Experimental Setup and Methods}

The experimental setup is a home-built sSNOM incorporated in the sample arm of a Michelson interferometer, as depicted schematically in Fig. 1. Light from a titanium:sapphire laser (Femtolasers Rainbow), with a spectral bandwidth of around $150 \mathrm{~nm}$ and centered at a wavelength of $820 \mathrm{~nm}$ (see the inset in the lower left of Fig. 1), is used to illuminate the sample. The power is set to $12 \mathrm{~mW}$ in front of the interferometer setup, and the direction of the electric field vector of the linearly polarized light can be adjusted using a half-wave plate (HWP). The light passes a beam splitter (BS) where $20 \%$ is reflected and is then focused on the sample using a reflective microscope objective

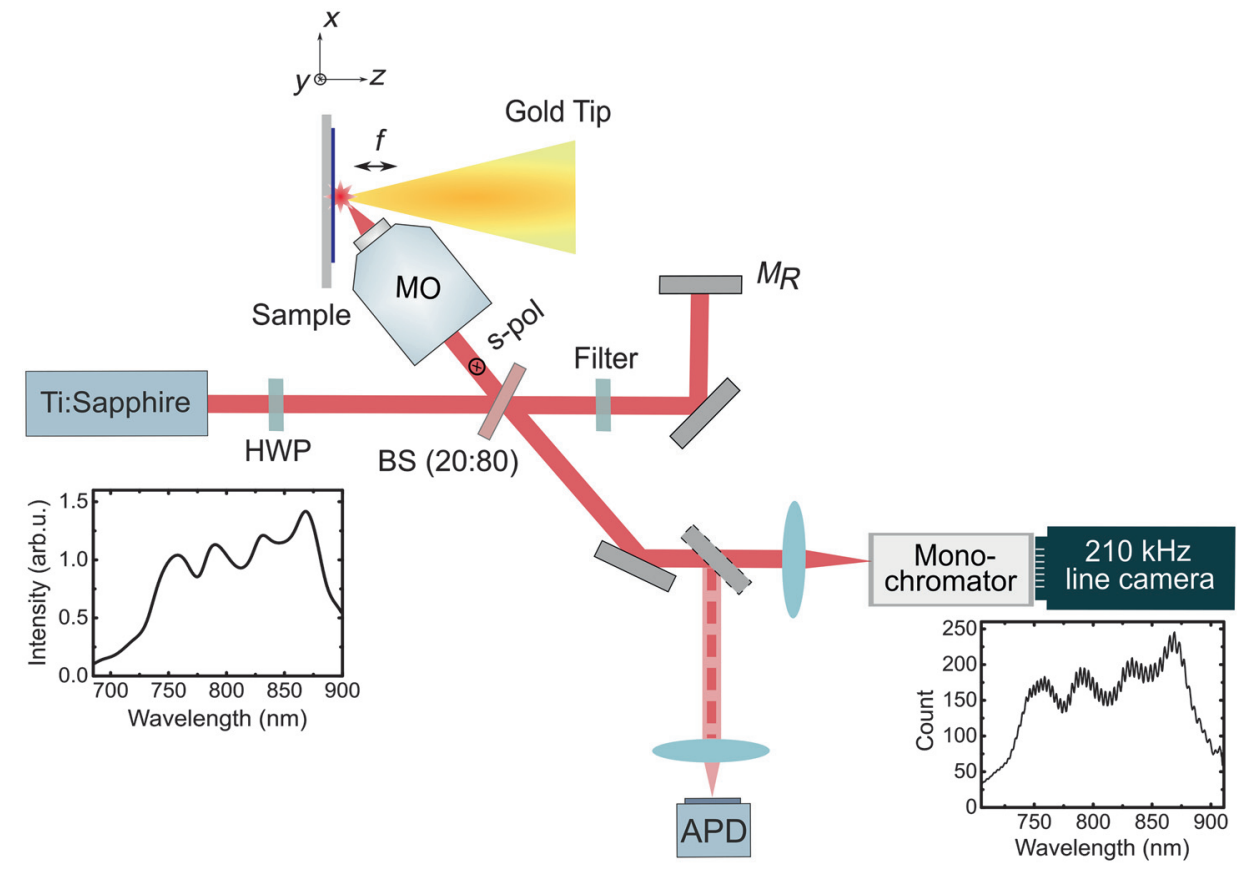

Fig. 1 Experimental setup for broad-bandwidth interference SNOM (BISNOM). Light from a broad-bandwidth titanium:sapphire laser (see the spectrum in the inset in the lower left) is focused onto the sample by an MO. The polarization is controlled by an HWP. A sharply etched gold tip is brought close to the sample to scatter light from the near-field region to the far field. The scattered light is collected by the MO. The larger part of the incident light $(80 \%)$ is transmitted through a BS to the reference arm of a Michelson interferometer, adjusted in power by a variable gray filter, reflected $\left(M_{R}\right)$, and superimposed with the light from the sample arm at the BS (see the spectral interferogram in the inset in the lower right). The light is detected either by an APD or by a monochromator followed by a fast CCD line camera. 
(MO, Beck Optronics Solutions, model 5003-000) with NA = 0.4 and with a working distance of $14.5 \mathrm{~mm}$. Using a reflective MO minimizes dispersion that would otherwise result in a varying fringe spacing in the recorded spectral interferograms. After the BS and the reflective MO, $<1 \mathrm{~mW}$ is incident on the sample and tip. For these input powers, laser-induced tip- and/or sample-heating effects were not observed. $80 \%$ of the incident light is transmitted at the BS and serves as a reference field $E_{R}$ in our measurements. For this, the power is adjusted by a variable gray filter before it is reflected back to the BS by a mirror $\left(\mathrm{M}_{\mathrm{R}}\right)$ that forms the reference arm of the Michelson interferometer. The reference arm length is adjusted to be shorter than the sample arm by $\sim 100 \mu \mathrm{m}$ in order to enable spectral interference with a convenient fringe spacing and is then kept constant during the measurement. To access the near-field region, a sharply etched gold nanotaper is brought in close proximity to the sample surface. Scattered light is collected in the backward direction by the MO. The part that is transmitted through the BS is overlapped with the reference field $E_{R}$, and the resulting superposition is collected for detection.

The near-field probe is a sharply etched single-crystalline gold nanotip with an apex radius of curvature of $\sim 10 \mathrm{~nm}$. The tip is brought in close vicinity to the sample surface and the tipsample distance is modulated at a frequency $f \approx 26 \mathrm{kHz}$ and with an amplitude of $12 \mathrm{~nm}$. The minimum tip-sample distance is kept at $4 \mathrm{~nm}$. Here, we set the light polarization to $s$, i.e., the electric field vector is along the $y$ axis such that it lies within the sample plane and is perpendicular to the tip axis.

We employ two different detection methods. In the first case, for quasimonochromatic measurements, the reference arm is blocked and the signal backscattered from the focus region is detected with an avalanche photodiode (APD, Hamamatsu, model C5331-02). It is further processed by a lock-in amplifier (Zurich Instruments HFLI), with the tip modulation frequency as the reference signal. The signal demodulated at the fundamental tip modulation frequency as well as those demodulated at its second, third, and fourth harmonics are obtained and stored. We label these spectrally unresolved signals produced by the lock-in amplifier with $S_{1 f}, S_{2 f}, S_{3 f}$, and $S_{4 f}$. In this case, with the reference arm blocked, the signal detected by the APD is constituted of light scattered out of the near field by the sharp tip, $E_{\mathrm{NF}}$, and of the unwanted background field $E_{B}$ scattered from the sample and from the tip shaft. Together, this results in a voltage proportional to $\left|E_{\mathrm{NF}}+E_{B}\right|^{2}$. Demodulating the signal at higher-order multiples of the tip modulation frequency makes the signal sensitive to the near-field contribution by recording an interference pattern between $E_{\mathrm{NF}}$ and $E_{B} \cdot{ }^{39}$

In the second case, for spectrally resolved measurements, the reference arm is unblocked and the output of the Michelson interferometer is steered to the monochromator (Princeton Instruments, IsoPlane-160) equipped with a fast line camera (e2V AViiVA EM4 with 512 pixels). The spectra measured now constitute a mixture of near field, background, and reference field:

$S(\lambda) \propto\left|E_{\mathrm{NF}}+E_{B}+E_{R}\right|^{2}$.

In front of the monochromator, the power detected from the sample arm alone amounts to $10 \mu \mathrm{W}$, and the power detected from the reference arm alone is $46 \mu \mathrm{W}$. The reference arm length is adjusted such that spectral interference fringes emerge with a spacing of 3 to $5 \mathrm{~nm}$ (see the inset at the lower right in Fig. 1). Equation (1) can be written as

$$
\begin{aligned}
S(\lambda) \propto & \left|E_{\mathrm{NF}}+E_{B}\right|^{2}+\left|E_{R}\right|^{2}+2 \operatorname{Re}\left[\left(E_{\mathrm{NF}}+E_{B}\right) \cdot E_{R}\right] \\
& \cdot \cos (k \cdot \Delta L),
\end{aligned}
$$

where the wavevector $k=2 \pi \lambda^{-1}$ and the path length difference between sample and reference arm is $\Delta L$. By separating the modulated part of the measured spectra, the term $\operatorname{Re}\left\{\left(E_{N F}+\right.\right.$ $\left.\left.E_{B}\right) \cdot E_{R}\right\}$ is isolated and the complexity of Eq. (2) is considerably reduced.

The remaining problem of suppressing $E_{B}$ with respect to $E_{\mathrm{NF}}$ is solved by the fast line camera behind the monochromator. The line camera has a maximum readout rate of $210 \mathrm{kHz}>8 \cdot f$, and we typically record 60,000 consecutive spectra with the maximum rate. In postprocessing, the recorded values are considered separately, i.e., for each wavelength component of the spectrum $S(\lambda)$, the line camera records a time series of 60,000 values. A Fourier series expansion is performed around multiples of the tip modulation frequency. For each pixel, the so-determined Fourier coefficients are quantities analogous to the signals generated by the lock-in amplifier in the monochromatic measurements. Assembling the respective Fourier components for all camera pixels results in spectra $S_{1 f}(\lambda)$ to $S_{4 f}(\lambda)$, demodulated at the first to fourth harmonic of the tip modulation frequency. ${ }^{44}$ As we will show below, for higher-order demodulation the background field $E_{B}$ is strongly suppressed in comparison with the near-field $E_{\mathrm{NF}}$, such that these spectra produce $\operatorname{Re}\left\{E_{\mathrm{NF}} E_{R}\right\}$.

We investigate individual antimony sulfide $\left(\mathrm{Sb}_{2} \mathrm{~S}_{3}\right)$ nanoparticles created on top of an $\mathrm{Sb}_{2} \mathrm{~S}_{3}$ film by EBL. ${ }^{9}$ Our samples are annealed at $300^{\circ} \mathrm{C}$ to form crystalline thin films and nanostructures. This procedure shifts the bandgap from 2.24 to $1.73 \mathrm{eV}$, and it reduces the above-gap absorption coefficient from $1.8 \times$ $10^{5} \mathrm{~cm}^{-1}$ at $450 \mathrm{~nm}$ to $7.5 \times 10^{4} \mathrm{~cm}^{-1}$ at $550 \mathrm{~nm}^{2}$ Here, we study their optical properties at wavelengths between 700 and $900 \mathrm{~nm}$, well below the bandgap. In this spectral region, $\mathrm{Sb}_{2} \mathrm{~S}_{3}$ has relatively low losses (absorption $<2 \times 10^{4} \mathrm{~cm}^{-1}$ at $800 \mathrm{~nm})^{2}$ and a high index of refraction, with a positive real part varying from 3.2 at $700 \mathrm{~nm}$ to 2.9 at $900 \mathrm{~nm}$, which means that, in our experiments, it can basically be considered as a lossless dielectric.

Here, we cover a flat, 140-nm-thick film of crystalline $\mathrm{Sb}_{2} \mathrm{~S}_{3}$ with $\mathrm{Sb}_{2} \mathrm{~S}_{3}$ nanoparticles, aiming at a cylindrical structure that should guide the incident light into the underlying thin film. The nanoparticles are created by $\mathrm{EBL}^{9}$ on top of the flat film in a regular order, with a spacing of $2 \mu \mathrm{m}$ [Fig. 2(a); more details on the sample preparation can be found in the Supplemental Materials]. The spacing is chosen sufficiently large to avoid dipolar couplings between adjacent nanoparticles such that each nanodot may be considered as an isolated nanoparticle. They are typically dome-shaped, with a round or slightly elliptical cross section of $\sim 350 \mathrm{~nm}$ diameter and a height of $\sim 150 \mathrm{~nm}$. Figure 2(a) shows a scanning electron microscope (SEM) image of the nanoparticles. A higher resolution image of an individual nanoparticle [Fig. 2(b)] reveals a slightly elliptical shape and a somewhat rough surface. The center of the particle seems to have collapsed to some extent after the annealing processes following EBL. These features (ellipticity, surface roughness, and indentation) vary slightly from one nanoparticle to the next. 

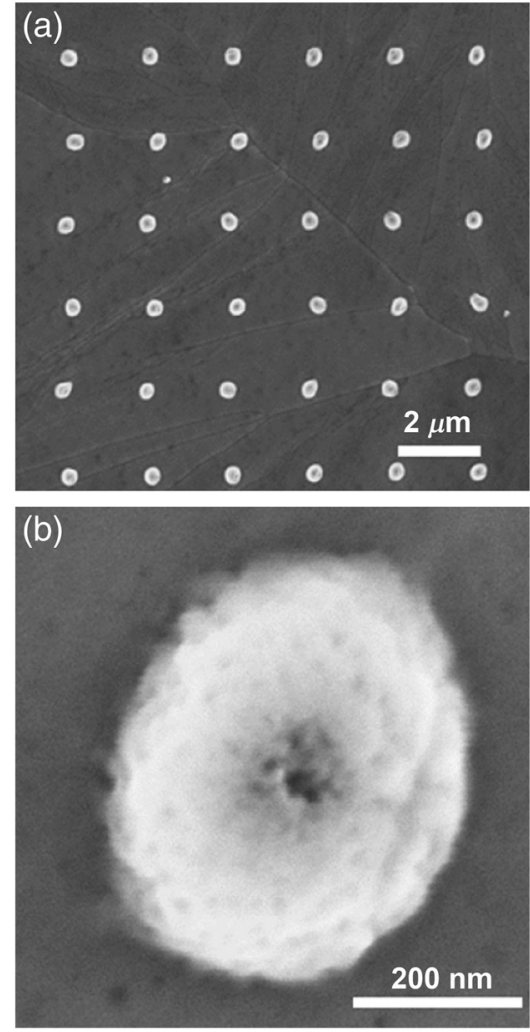

Fig. $2 \mathrm{SEM}$ images of the sample. (a) $\mathrm{Sb}_{2} \mathrm{~S}_{3}$ nanodots are created on a flat, compact $\mathrm{Sb}_{2} \mathrm{~S}_{3}$ film by EBL. The nanodots are regularly spaced by $2 \mu \mathrm{m}$, and their shape varies slightly. (b) The SEM image of an individual nanodot shows a slight ellipticity and a dark shadow in the center where the material has collapsed after EBL and annealing.

\section{Results and Discussion}

Before recording the BISNOM spectra, we perform preparatory experiments to validate the near-field contrast and spatial resolution of the optical signals in our near-field microscope. For this, we use a quasimonochromatic spectrum for excitation, created by inserting a 40-nm bandwidth interferometric filter centered at a wavelength of $900 \mathrm{~nm}$ in front of the BS, and the APD and lock-in amplifier for detection. The demodulated signals $S_{1 f}, S_{2 f}, S_{3 f}$, and $S_{4 f}$ are generated by the lock-in amplifier and are recorded during the measurement.

Figure 3(a) shows approach curves recorded above a flat $\mathrm{Sb}_{2} \mathrm{~S}_{3}$ film, i.e., the demodulated signals as a function of tip-sample distance. The approach is stopped when the tuning fork oscillation amplitude is reduced by $2.5 \%$ [see the inset of Fig. 3(a)]. This position, which corresponds to a minimum tip-sample distance of about $4 \mathrm{~nm}$, is later taken as the set point for lateral sSNOM scans. The blue, green, and red curves in Fig. 3(a) show the optical signals $S_{1 f}, S_{2 f}$, and $S_{4 f}$, respectively, when the sample is retracted from the tip. To retain clarity in Fig. 3(a), $S_{3 f}$ is not shown, as its shape is very similar to that of $S_{2 f}$. As the tip is retracted, the signal amplitude decays quickly. This is reflected by all three curves shown in Fig. 3(a), where the strong peaks at the minimum tip-sample distance of $4 \mathrm{~nm}$ decrease quickly with increasing tip-sample distance. Compared to the near field, the background field's dependence on the tip-sample distance is weaker. As a result, the first-order demodulated signal $S_{1 f}$, which mainly probes background scattering from the tip shaft, remains at a rather high level for large tip-sample distances. Fluctuations arise from finite mechanical instabilities and/or laser noise. The higher demodulation orders, however, measure an interference between the near-field signal and the background. Here, a measurable signal is only seen for tip-sample distance of $<10 \mathrm{~nm}$. The signal amplitude in contact decreases when going from $2 f$ to $4 f$ due to the demodulation of the signal recorded with finite tapping amplitudes. ${ }^{41}$

To further evaluate the optical image capabilities of the nearfield microscope, we then scanned a single $\mathrm{Sb}_{2} \mathrm{~S}_{3}$ nanodot with the quasimonochromatic measurement method while the tuning fork amplitude was kept constant. The heights of the sample and the demodulated signals $S_{1 f}$ to $S_{4 f}$ were recorded over an area of $1 \mu \mathrm{m}$ by $1 \mu \mathrm{m}$. The sample was scanned linewise in the $x$ direction with a step size of $4 \mathrm{~nm}$ and with a step size of $16 \mathrm{~nm}$ between the lines, i.e., in the $y$ direction. The topographic image displayed in Fig. 3(b) shows that this nanodot was almost circular, with lengths of 360 and $380 \mathrm{~nm}$ of the short and the long axis of an ellipse, respectively, and with a height of about $150 \mathrm{~nm}$. The simultaneously recorded maps of the signals $S_{1 f}$, $S_{2 f}$, and $S_{4 f}$ are displayed in Figs. 3(c)-3(e), respectively. They all display a clear optical signal drop in the shape of a ring over the particle. In the following, we investigate several of the observed features one by one.

The near-field images of the nanodot in Fig. 3 show a rather smooth surface and sharp signal change at the edge of the nanoparticle. The maps of the $S_{1 f}$ and $S_{2 f}$ signals display some variation across the surrounding $\mathrm{Sb}_{2} \mathrm{~S}_{3}$ film, which may be caused by residual background interference. The $S_{1 f}$ and $S_{2 f}$ maps both show a strong and sharp signal increase at the left-hand edge of the nanoparticle, which is probably caused by the grazing incidence of the illuminating laser light. The spatial resolution of the optical images is better than $20 \mathrm{~nm}$ (see the Supplemental Materials).

The best near-field contrast is reached with the $S_{4 f}$ signal [Fig. 3(e)]. The optical signal measured across the particle is reduced in the ring-shaped area with respect to the signal measured on the film by roughly $90 \%$. This difference can be understood as follows. While the tip is within a small distance to the flat film, incident light and light reflected from the film couple to the polarizability component of the tip dipole that is oriented along the polarization direction of the incident laser field. The laser is polarized perpendicular to the tip axis to avoid strong resonant excitation of the longitudinal tip polarizability. The $y$-polarized tip dipole induces an image dipole in the sample and the near-field coupling between the tip dipole and the image dipole enhances the field that is scattered from the tip to the detector. In the flat film region, the near-field contrast thus results from the coupling of the tip and image dipoles, as introduced by Keilmann. ${ }^{40}$ For such a planar sample, the LDOS is spatially and spectrally homogeneous, and the near-field signal probes the dielectric function of the bulk material. ${ }^{39,40}$ As the tip reaches the edge of the particle, it no longer couples to an extended film, but to a nanoparticle of finite size. Hence, the LDOS of the sample is dominated by the resonant optical modes of the nanoparticle. As will be discussed in more detail below, the SNOM images essentially provide a spatial map of the profile of these modes.

We find that the near-field signal rises steadily from the low-signal region near the particle rim toward the center of the 

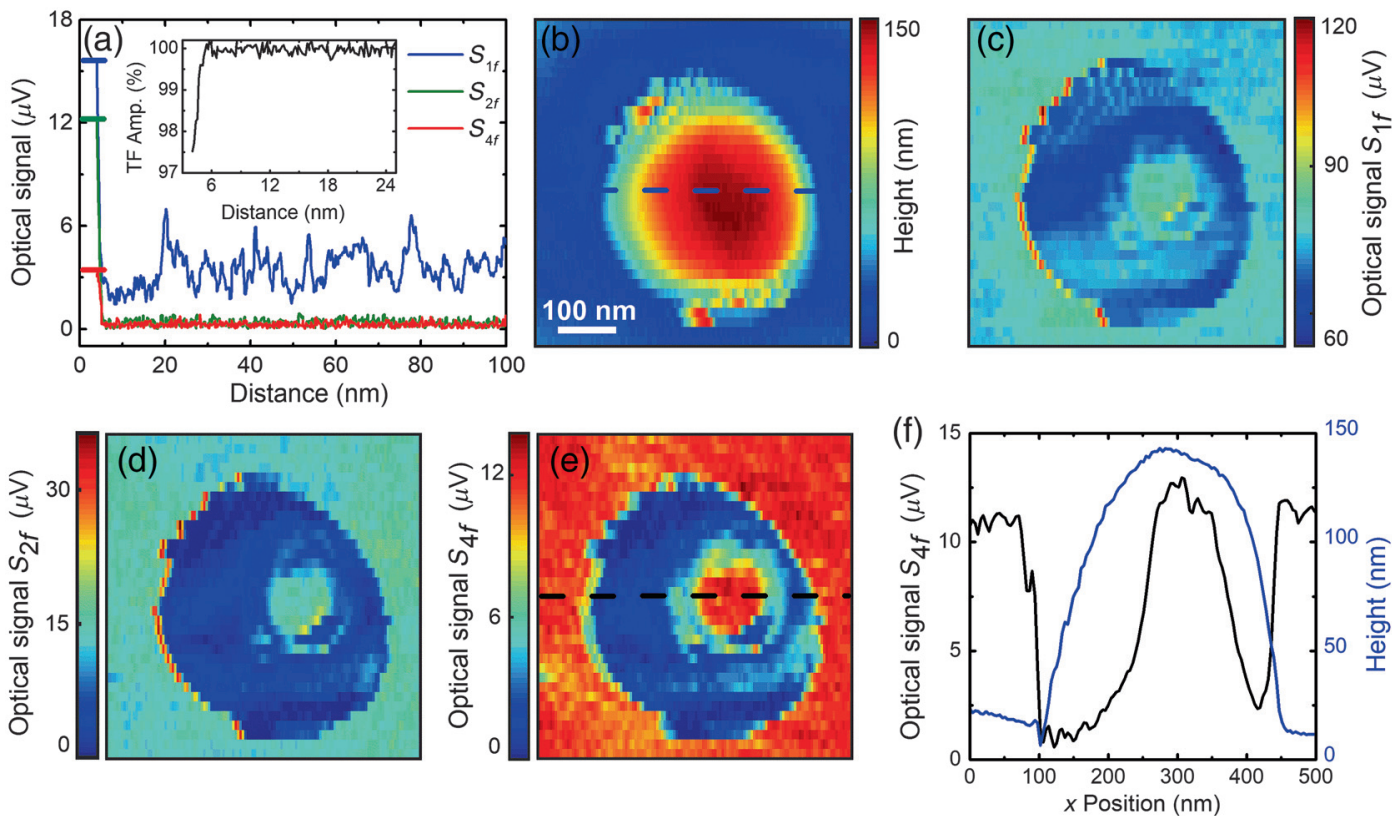

Fig. 3 Quasimonochromatic SNOM measurements of an individual nanoparticle using a 40-nm bandwidth laser spectrum centered at $900 \mathrm{~nm}$ for excitation and the APD and lock-in amplifier for detection. (a) Optical signal demodulated at the first (blue curve), second (green curve), and fourth harmonic (red curve) of the tip modulation frequency as a function of tip-sample distance. The higher-order demodulated signals demonstrate an improved near-field contrast. The inset shows the simultaneously recorded tuning fork amplitude. (b) A topographical map and (c)-(e) maps of the optical signals $S_{1 f}, S_{2 f}$, and $S_{4 f}$, respectively. All maps show a ring-shaped intensity distribution. (f) Cross cuts through the topographical map (blue curve) and the $S_{4 f}$ signal (black curve) along dashed lines in (b) and (e).

nanodot. The over-all symmetry is roughly radial. To show this more clearly, cuts through the topographic image and through the $S_{4 f}$ map [along the dashed lines in Figs. 3(b) and 3(e)] are plotted together in Fig. 3(f). The topography contour (blue curve) is a smooth cone, with its maximum leaning somewhat to the right. We thus interpret the central feature in the SNOM images as the spatial extent of the optical modes of the nanoparticle that are excited by the external laser light and probed by their coupling to the near-field tip. The dome-like shape of the observed mode is reminiscent of fundamental fiber-optical modes and suggests that waveguide modes that are excited in the $\mathrm{Sb}_{2} \mathrm{~S}_{3}$ nanoparticle are of a similar shape.

In order to investigate the nanodot modes further, we calculate the solutions of the vector Helmholtz equation for a weakly guiding, cylindrical waveguide, as it is routinely applied to fiber-optical waveguides. ${ }^{45,46}$ Taking a long cylinder of $200 \mathrm{~nm}$ radius, the refractive index of $\mathrm{Sb}_{2} \mathrm{~S}_{3} n_{1}=3.04$ at a wavelength of $800 \mathrm{~nm}$, and $n_{2}=1$ for the surrounding medium air, there exist four solutions for guided waves. Adopting the terminology that was introduced for fiber modes in 1971 by Gloge, ${ }^{45}$ these are the $\mathrm{LP}_{01}, \mathrm{LP}_{11}, \mathrm{LP}_{02}$, and $\mathrm{LP}_{21}$ modes, which are strongly localized within the waveguide. Here, the first index indicates the number of pairs of nodes of the electric field strength in the azimuthal coordinate, and the second index gives the number of nodes in the radial direction, including the approach toward zero for large radii.

We assume that the LDOS in the nanodot region is mainly given as a sum over these bound modes with eigenvectors $\vec{e}_{m}(\vec{r})$ and eigenfrequencies $\omega_{m} \cdot{ }^{26}$ Each of these modes is characterized by a finite damping rate $\gamma_{m}$ that describes the temporal decay of the electromagnetic field emitted by this mode after impulse excitation. In the limit of sufficiently weak damping, the LDOS may be phenomenologically written as

$\rho(\vec{r}, \omega)=\sum_{m}\left|\vec{e}_{m}(\vec{r}, \omega)\right|^{2} \delta_{g}\left(\omega-\omega_{m}\right)$.

Here, $\delta_{g}\left(\omega-\omega_{m}\right)=\frac{-2 i \omega_{m}^{\prime}}{\pi\left(\omega_{m}^{2}-\omega^{2}-2 i \omega \gamma_{m}\right)}$ with $\omega_{m}^{\prime}=\sqrt{\omega_{m}^{2}-\gamma_{m}^{2}}$ is a generalized Dirac delta function. Its integral along the frequency axis gives unity. Quite generally, the damping can be induced by the finite lifetime of the mode $m, T 1_{m}$, and possible pure dephasing processes with pure dephasing time $T 2_{m}^{*}$ resulting from stochastic fluctuations of the environment. Phenomenologically, the damping rate can then be taken as $\gamma_{m}=\left(2 T 1_{m}\right)^{-1}+\left(T 2_{m}^{*}\right)^{-1}$ and the mode can be characterized by a complex resonance frequency $\tilde{\omega}_{m}=\omega_{m}-i \gamma_{m}$. The contribution of unbound radiation modes is neglected since their overlap with the nanodot core is small. For the perfect cylindrical waveguide considered here, for each bound mode a continuum of modes that are propagating along the fiber axis exists and the LDOS is spectrally flat.

The LDOS for a cylindrical waveguide of $200 \mathrm{~nm}$ radius is plotted in Fig. 4(a) for a wavelength of $800 \mathrm{~nm}$. In this picture, the corresponding eigenvectors are twofold polarization degenerate for light field polarization along the $x$ and $y$ axes, while the $z$ axis is the direction of propagation of the guided waves. An SNOM measurement carried out by scanning a point dipole 

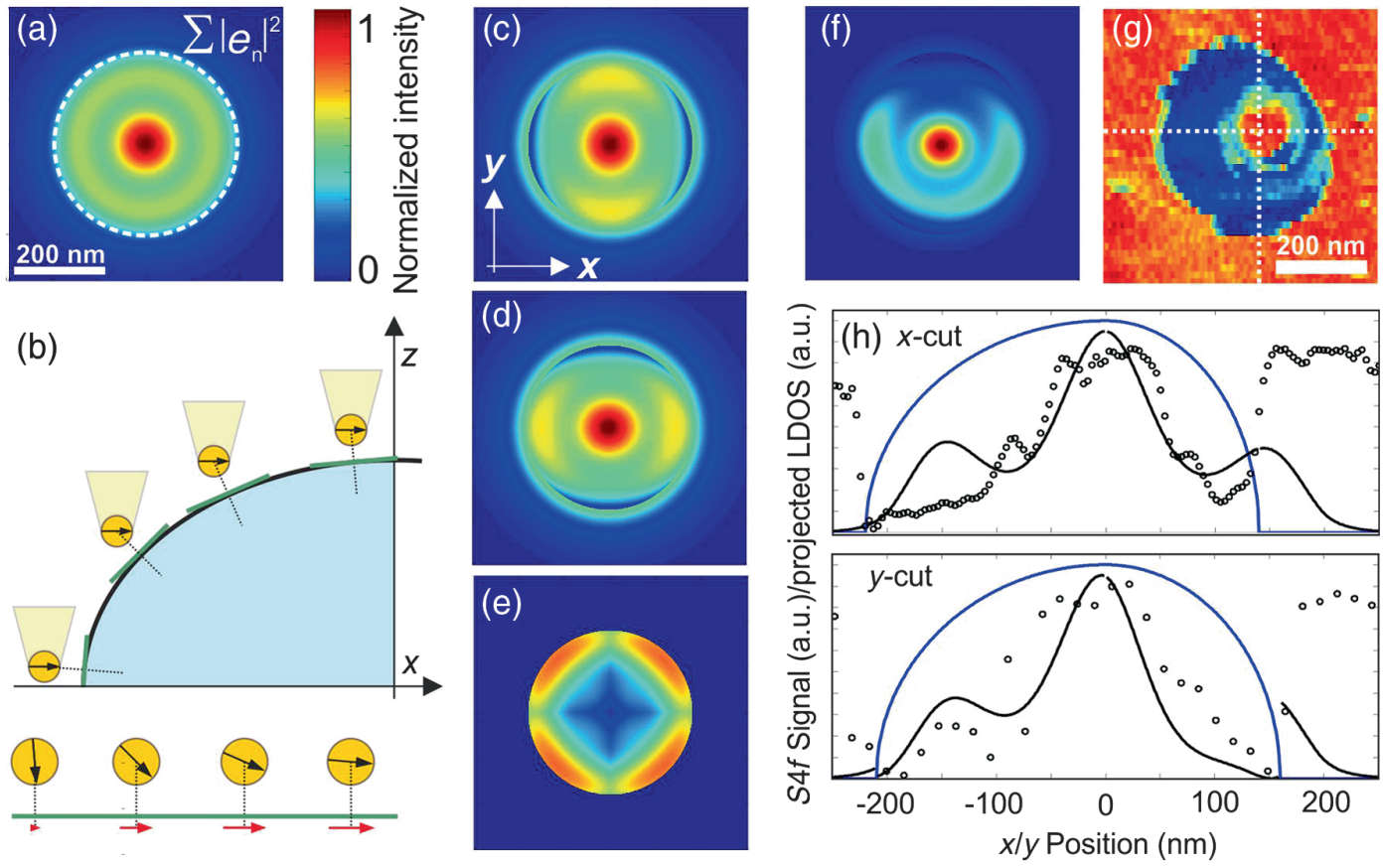

Fig. 4 (a) Local density of states in an $\mathrm{Sb}_{2} \mathrm{~S}_{3}$-nanodot with a circular cross section of a 200-nm radius at a wavelength of $800 \mathrm{~nm}$. (b) An $x$-oriented tip dipole is moved along the $x$ axis from the left rim to the center over the surface of the elliptical nanodot. Below is shown how the dipole orientation changes relative to the surface, and the red arrows at the bottom indicate the projection on the surface. (c) The projected LDOS as probed with an $x$-oriented tip dipole shows minima at the left and right rims, corresponding to the small projection of the tip-dipole onto the surface at these edges. (d) Using a $y$-oriented tip dipole results in a 90-deg rotated image, and (e) a $z$-oriented dipole particularly probes the edge of the rim. (f) The asymmetric SNOM map calculated for a wavelength of $800 \mathrm{~nm}$ and $(\mathrm{g})$ for comparison, the measured $S_{4 f}$ map [same as Fig. 3(e)]. The main maximum as well as the asymmetric side lobes on the left and lower right and the near-zero signal regions at the top and bottom are well recreated. (h) Horizontal and vertical cuts (upper and lower graphs, respectively) of the measured SNOM map (black circles) together with the calculated SNOM signals (black curve), calculated for the nanodot curvature shown by the blue curves.

$\vec{p}$ oriented along $\hat{u}$ across a structure with localized modes probes the projected LDOS, ${ }^{13,26}$

$\rho_{\hat{u}}(\vec{r}, \omega)=\frac{2 \omega}{\pi c^{2}} \operatorname{Im}[\hat{u} \cdot \stackrel{\leftrightarrow}{G}(\vec{r}, \vec{r}, \omega) \cdot \hat{u}]$

where, for weakly dissipative systems, Green's function can be written as $^{26}$

$\stackrel{\leftrightarrow}{G}\left(\vec{r}, \vec{r}^{\prime}, \omega\right) \approx c^{2} \sum_{m} \frac{\vec{e}_{m}^{*}\left(\vec{r}^{\prime}\right) \otimes \vec{e}_{m}(\vec{r})}{\omega_{m}^{2}-\omega^{2}-2 i \omega \gamma_{m}}$.

In principle, if a tip closely representing a point dipole was scanned across a flat surface with such a waveguide mode profile (such as the flat top of a pillar of $\mathrm{Sb}_{2} \mathrm{~S}_{3}$ surrounded by air), the resulting SNOM map should be an image of the calculated LDOS shown in Fig. 4(a). In our experiment, however, the surface of the nanodot is not flat, but it is more truthfully described by a flattened half-ellipse with circular base of 200-nm radius and a shorter height of $140 \mathrm{~nm}$. When scanning a point dipole across the nanodot, the point dipole projection onto its surface changes continuously. We have calculated the projection of a tip dipole $\vec{p}_{\text {tip }}$ of a fixed orientation onto the surface as a function of the tip position $\vec{r}$ and have substituted the point dipole orientation $\hat{u}$ in Eq. (4) by this projection (more information on these calculations can be found in the Supplemental Materials). Figure 4(b) shows in a sketch how the projection onto the surface (the red arrow) increases as an $x$-oriented tip dipole is moved along the central $x$ axis from the rim of the particle to its center. When scanning an $x$-oriented dipole along the central $y$ axis, however, the projection onto the surface does not change. In total, the projected LDOS then resembles the LDOS shown in Fig. 4(a) but has minima at the left and right sides [see Fig. 4(c)]. Analogously, when scanning a $y$-oriented dipole across the nanodot, the projected LDOS appears rotated by $90 \mathrm{deg}$ [Fig. 4(d)]. In contrast, a z-oriented tip dipole creates the largest projection close to the particle rim, resulting in the image shown in Fig. 4(e).

In our experiment, the light was incident from a direction in the $x-z$ plane, with an angle of $\sim 20$ deg to the negative $x$ axis and $\sim 70$ deg to the $z$ axis, with the field polarization along the $y$ axis. However, as the tips are etched and then glued onto the tuning fork manually, their orientation relative to the sample is with some error margin $(\sim 10 \mathrm{deg})$. Thus, an excitation of the $z$-oriented as well as the $x$-oriented tip dipoles can hardly 
be avoided due to uncertainties in the alignment of the tips, and an excitation of the three components on the diagonal of the polarizability tensor was considered. Finally, the radiation of the tip dipole, locally enhanced by its coupling to the waveguide modes into the far field (i.e., the detector, located at position $\vec{R}_{\text {det }}$ in the direction of the incident light), is calculated to yield the simulated SNOM map shown in Fig. 4(f). The SNOM map displays a distinct radial asymmetry, which is likely to arise from the particular orientation of the tip dipole. The simulation using the projection of the tip dipole on an elliptical surface explains in particular the rather narrow central maximum in the SNOM map as well as the side lobes to the left and lower right and the near-zero signal strength at the upper and lower rim [compare $S_{4 f}$ map in Fig. 4(g)]. Figure 4(h) compares the simulated (black curves) and measured $S_{4 f}$ signals (black circles) along a horizontal and a vertical cut (upper and lower graph, respectively). These cuts have been calculated for the LDOS shown in Fig. 4(a), but for differently curved surfaces (indicated by the blue curves), in order to account for the slightly asymmetric shape of the nanoparticle. One can see that neither simulated nor measured values reach zero at the left and right rim of the nanodot (see $x$-cut), but they do so at the upper and lower rim ( $y$-cut). Even though the simulations reproduce most of the experimental observations, some minor discrepancies remain. Specifically, the plateau formed around the center of the measured SNOM map and a very sharp ring around this central plateau are not well reproduced. These differences may be the effect of the finite disorder seen in Fig. 2(b) on the local field enhancement. In summary, the simulations show that the shape of the SNOM images resembles the projected LDOS given by the bound modes of a circular waveguide with a dome-like surface topography.

In the following, we will employ spectrally resolved nearfield measurements of an $\mathrm{Sb}_{2} \mathrm{~S}_{3}$ nanodot to study these modes in more detail. We can approach this in two ways. First, the waveguide modes proposed above provide a solution for a continuum of wavelengths. At a fixed spatial position, these modes can be excited across the complete spectral range covered by the broad-bandwidth laser, and the scattered spectra therefore should show negligible spectral variation in our experiments. This is different in case that resonant modes of the nanodot are excited, showing distinct spectral resonances. We will next present spectra recorded at different positions on the nanodot and the film to distinguish between both cases.

In order to verify that the BISNOM measurements probe a pure near-field contrast, we record near-field spectra during an approach over the flat $\mathrm{Sb}_{2} \mathrm{~S}_{3}$ film. Figures 5(a) and 5(b) compare the spectra demodulated at the fundamental tip modulation frequency $S_{1 f}(\lambda)$, and spectra demodulated at the fourth harmonic, $S_{4 f}(\lambda)$, for a few tip-sample distances between 3 and $12 \mathrm{~nm}$. The $S_{1 f}(\lambda)$ spectrum [Fig. 5(a)] increases in intensity when the sample is removed from the tip, and the spectral shape changes. Both effects can be ascribed to interference of the reference field with a strong background signal scattered from the tip. In contrast, the $S_{4 f}(\lambda)$ spectrum [Fig. 5(b)] decays strongly as the sample is removed from the tip; as the distance is increased by $12 \mathrm{~nm}$, the signal mostly vanishes. During the process, the spectral shape is retained. In order to show that the measured $S_{4 f}(\lambda)$ spectrum represents in good approximation the near-field spectrum, we have calculated the latter.

We model the tip polarizability in the $x$ and $y$ directions (perpendicular to the tip axis) by the polarizability of a gold sphere with radius $R=10 \mathrm{~nm}$. A dipole oscillation is excited by an incident field polarized in the $y$ direction. Following Knoll and Keilmann, ${ }^{40}$ the tip dipole causes a polarization in the material. The field emitted by the image dipole acts back on the tip dipole, effectively enhancing the incident field. This is described by an effective polarizability, which increases in a super-linear fashion as the tip-sample distance is decreased. In Fig. 5(c), the $y y$ matrix element of the effective polarizability (the only nonzero element under excitation with $y$-polarized light) is plotted as a function of wavelength for the same tip-sample distances $d$ as were measured in the experiment and taking into account the dielectric functions of gold and of $\mathrm{Sb}_{2} \mathrm{~S}_{3}$. One can see that the effective polarizability increases as the tip-sample distance decreases. The increase toward short wavelengths is caused by the tip resonance, which is far detuned to the blue. This spectral shape is preserved during the approach. We calculate the dipole field component that is radiated toward the detector and mix it with the reference field to yield the nearfield spectrum $S_{\mathrm{NF}}(\lambda, d)$ for the different distances $d$. Here, we have used the measured reference spectrum [shown in the inset of Fig. 5(d)] as both the reference and incident spectrum. The result is shown in Fig. 5(d). The agreement with the measured $S_{4 f}$ spectra is excellent, both in spectral shape and in distance dependence.

We now perform a spectrally resolved BISNOM scan of an $\mathrm{Sb}_{2} \mathrm{~S}_{3}$ nanoparticle using the monochromator and the fast line camera. The laser spectrum used for this particular measurement is the one shown in the setup sketch in Fig. 1. We record complete near-field spectra $S_{1 f}(\lambda), S_{2 f}(\lambda), S_{3 f}(\lambda)$, and $S_{4 f}(\lambda)$ across a 500-nm by 400-nm large area, and with a step size of 12.5 and $20 \mathrm{~nm}$ in the $x$ and $y$ directions, respectively. In Figs. 6(a)-6(c), we show sets of these four spectra exemplarily for three different positions: in the center of the nanoparticle [Pos. 1, Fig. 6(a)], on the flat $\mathrm{Sb}_{2} \mathrm{~S}_{3}$ film [Pos. 2, Fig. 6(b)], and on the outer area of the nanoparticle where the near-field signal is strongly reduced [Pos. 3, Fig. 6(c)]. The positions are marked in the SNOM map in the inset in Fig. 6(d), which has been prepared by plotting the spectrally integrated signal $S_{4 f}(\lambda)$ as a function of position on the sample. Within each of the Figs. 6(a)-6(c) one can see that the measured $S_{2 f}(\lambda), S_{3 f}(\lambda)$, and $S_{4 f}(\lambda)$ spectra closely resemble each other, while the spectral shape of $S_{1 f}(\lambda)$ clearly deviates from these and furthermore varies between the positions. In agreement with the observation shown in Figs. 5(a) and 5(b), this leads us to conclude that the higher-order demodulated spectra reliably reflect the near-field spectra, while spectral interference of the background and reference fields leads to spectral variations in the $S_{1 f}(\lambda)$ signal. In contrast, the higher-order demodulated spectra seem to hardly vary as the position on the sample is changed. In order to facilitate a direct comparison, the $S_{4 f}(\lambda)$ spectra of the three positions are plotted together in Fig. 6(d). The black curve represents a near-field spectrum calculated from the effective polarizability above a flat $\mathrm{Sb}_{2} \mathrm{~S}_{3}$ film and using the laser input spectrum shown in Fig. 1. As expected, the $S_{4 f}(\lambda)$ spectrum recorded on the film (Pos. 2, dotted red curve) matches the calculated spectrum well. A similar agreement can be seen for the $S_{4 f}(\lambda)$ spectrum recorded at the center of the nanodot (Pos. 1, dashed red curve). This supports our interpretation of the field profile as that of a mode, which has a broad bandwidth and is spectrally flat within our measurement range. Coupling to the nanodot is nearly wavelengthindependent, which is reflected by the close resemblance of the calculated spectra and those measured on the film and 

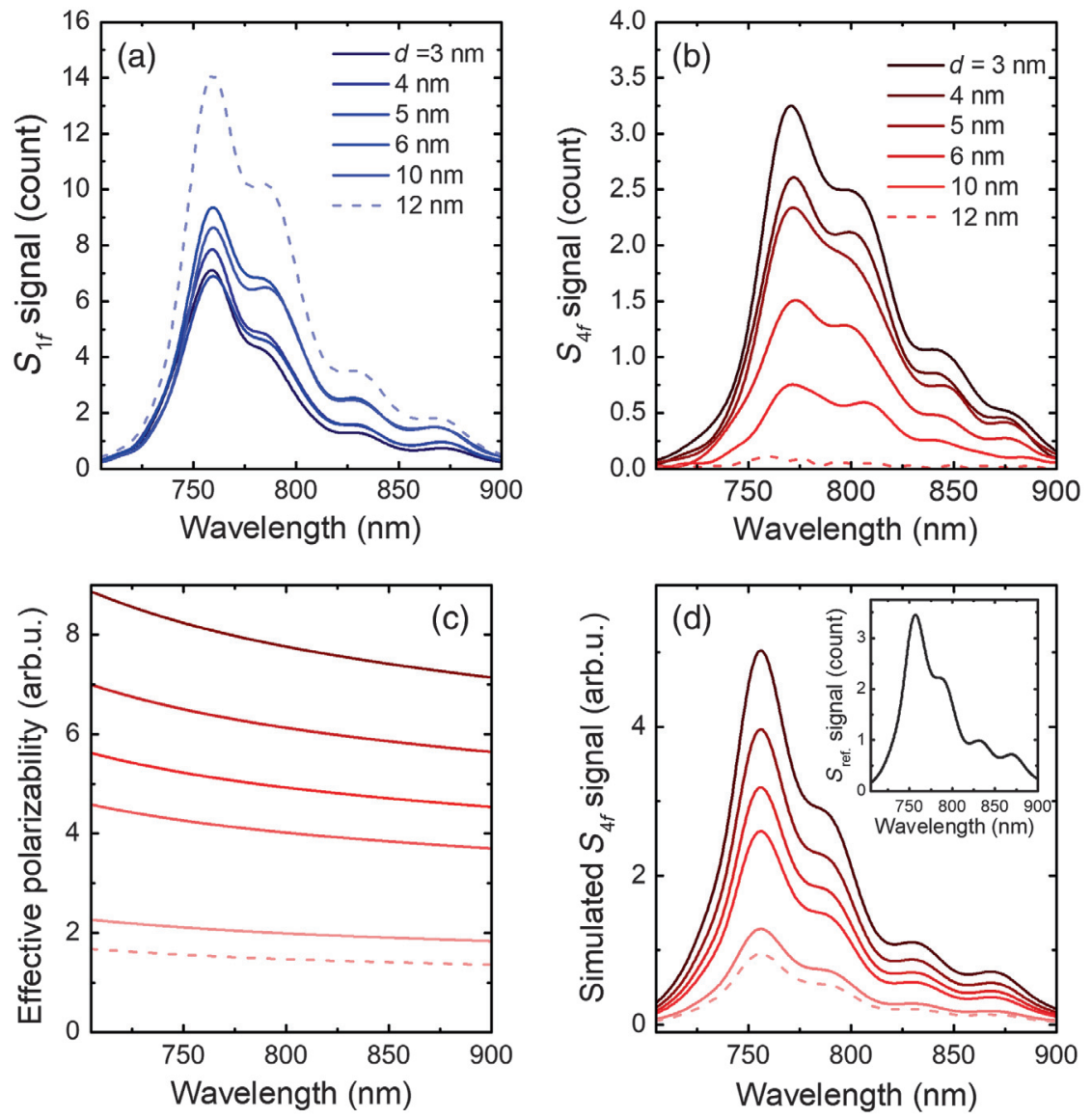

Fig. 5 Spectrally resolved approach curves measured above the flat $\mathrm{Sb}_{2} \mathrm{~S}_{3}$ film. (a) The spectra recorded on the $\mathrm{Sb}_{2} \mathrm{~S}_{3}$ film, demodulated at the fundamental tip modulation frequency $S_{1 f}(\lambda)$ as a function of the tip-sample distance $d$. Due to background interference, the spectra change their shape as the tip is retracted. (b) The signal demodulated at the fourth harmonic $S_{4 f}(\lambda)$ retains its shape and decreases rapidly as the tip-sample distance increases. (c) The effective tip polarizability calculated for the same tip-sample distances as used in the measurements; and (d) the calculated near-field spectra. The inset shows the reference spectrum that was measured in the experiment and used as the input spectrum for the simulations. The calculated near-field spectra are in good agreement with the measured $S_{4 f}(\lambda)$ spectra.

the central area of the nanodot. The last spectrum has been measured in the outer area of the nanodot, where the over-all signal strength is markedly reduced (Pos. 3, solid red curve). For a better comparison with the other two spectra, this one is scaled by a factor 2.2. The slightly differing shape can thus be attributed to the lower signal-to-noise ratio at this position. Together, the measurements presented in Fig. 6 verify the assumption of the broad bandwidth and flat spectral shape of the observed fundamental optical mode of the nanodot. The spatial-spectral behavior is similar to that of guided wave modes. No evidence for resonant modes of the nanodot is found.

As a final verification of the spatial-spectral mode properties, we extract spectral near-field maps from the BISNOM data. Since the waveguide modes are spectrally flat, we expect very similar near-field maps for different spectral bands, possibly increasing slightly in diameter with increasing wavelength, as known from fiber modes. In Figs. 7(a)-7(g), we have plotted maps created by spectrally integrating the $S_{4 f}$ signal over 30$\mathrm{nm}$ wide spectral bands, centered at equally spaced wavelengths from 715 to $895 \mathrm{~nm}$. The contrast in these SNOM maps remains high throughout these seven spectral bands, which is yet another implication of the negligible spectral variation of the waveguide modes. At the short- and the long-wavelength edges, the measurement noise is increased due to the lower spectral power density of the laser. In each of these maps, a ring-shaped signal decrease can be seen clearly. On closer inspection, the characteristic features from the projected LDOS calculation can be found here again, namely the secondary maxima at the left and lower right of the nanodot, and the near-zero signal regions at the top and bottom. Compared to the earlier investigated nanodot (cmp. Fig. 4), here the main maximum appears to be more centered, which indicates that the nanodot is more regularly shaped.

Figure 7(h) compares cross cuts through the maps created at the outermost spectral bands at 715 and $865 \mathrm{~nm}$ [Figs. 7(a) and 7(f), respectively] with calculated SNOM measurements above an elliptical surface over the $\mathrm{LP}_{01}, \mathrm{LP}_{11}, \mathrm{LP}_{02}$, and $\mathrm{LP}_{21}$ mode profiles, once calculated for a wavelength of $715 \mathrm{~nm}$ and once for $865 \mathrm{~nm}$. The measured values are shown as symbols in Fig. 7(h) and the calculated values as solid curves. As all curves 

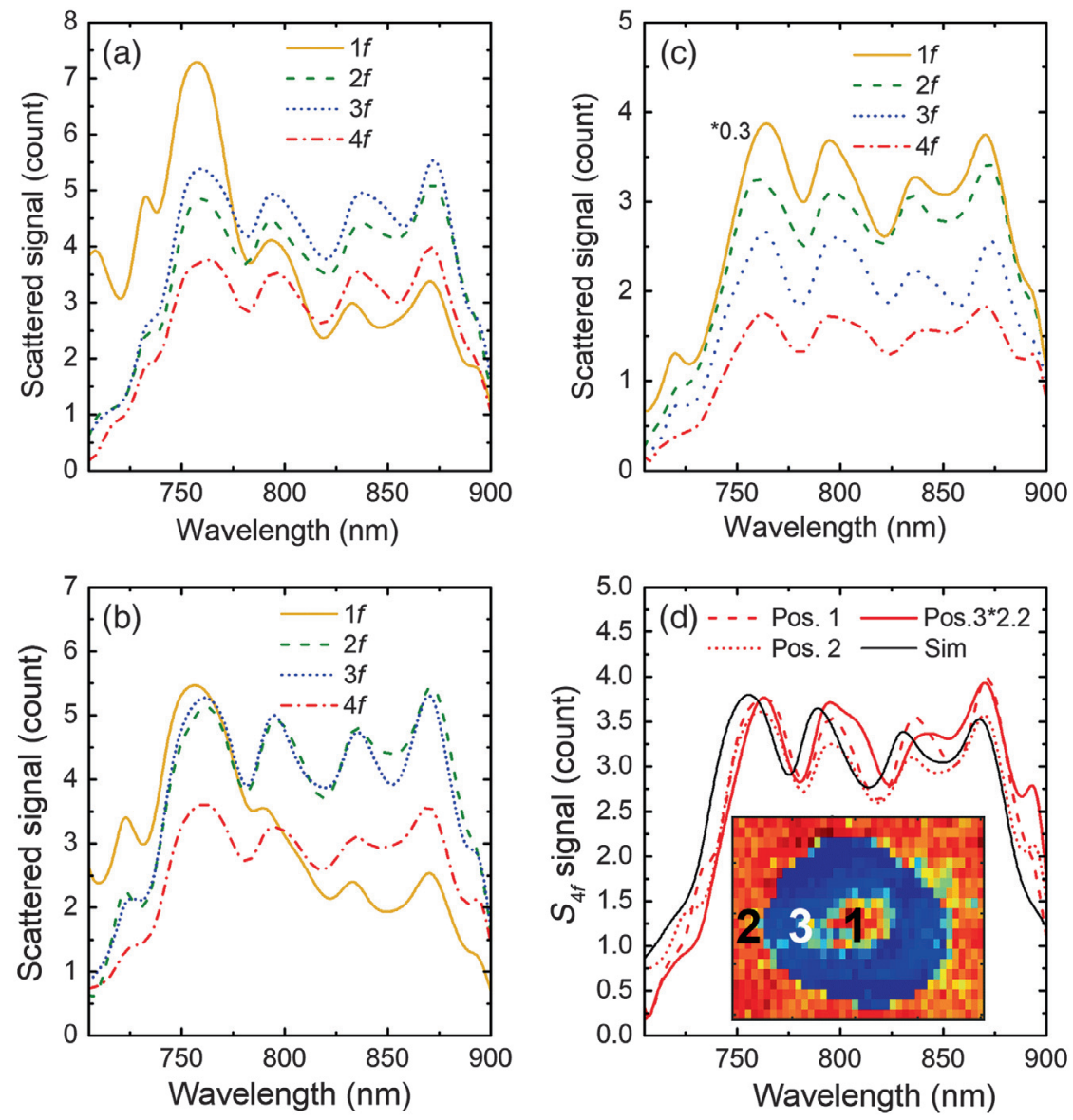

Fig. 6 Near-field spectra recorded at different positions on an $\mathrm{Sb}_{2} \mathrm{~S}_{3}$ nanoparticle using the monochromator and fast line camera. (a) The demodulated signals $S_{1 f}(\lambda)$ to $S_{4 f}(\lambda)$ recorded with the tip in close contact above the center of the nanodot; (b) on the film; and (c) above the outer area on the nanodot. (d) Comparison of the three $S_{4 f}(\lambda)$ spectra shown in (a)-(c) and a calculated $S_{4 f}$ spectrum (black curve). The inset indicates the three positions where the spectra shown in this figure are recorded.

are normalized, the larger extent of the long-wavelength mode is reflected by the slower decrease with a larger radius (red curve). The general shape of the cross cuts through the measured nearfield maps agrees well with the simulated projected LDOS. The increase in diameter with wavelength predicted by the modeling cannot easily be seen in the measurements. Seeing this subtle effect is certainly made difficult by the finite signal-to-noise ratio measurement. In addition, it may be obscured by the effect of the structural disorder of the nanodot on its optical eigenmodes. In particular, the imperfections in the center of the dot seen in Fig. 2(b) are likely to affect the mode profile structure near the surface. Such disorder effects are likely to be seen on the left part of the nanodot center. Together, the spectrally resolved near-field measurements presented in Figs. 6 and 7 convincingly demonstrate spatial mapping of the mode profiles of the individual $\mathrm{Sb}_{2} \mathrm{~S}_{3}$ nanodot. They show that the spatial mode profiles of these dots are similar to those of the lowest-order LP guided modes of the idealized cylindrical waveguide. No evidence for resonant cavity modes of the nanodot is found in these measurements. This suggests that backreflections at the interface between the nanodot and the supporting $\mathrm{Sb}_{2} \mathrm{~S}_{3}$ film are too weak to result in Fabry-Perot-like standing modes of the dielectric nanodot cavity.

\section{Summary and Conclusions}

We have realized a new interferometrically detected scanning near-field optical spectroscopy technique with a broad bandwidth in the near-infrared. The key to this method is a fast CCD line camera capable of recording complete spectra with a readout rate of more than eight times the tip modulation frequency such that near-field spectra demodulated at the fourth order can be obtained in postprocessing. Furthermore, we have incorporated the near-field microscope in a Michelson interferometer for homodyne mixing and boosting the weak near-field signal.

We have demonstrated the potential of broad-bandwidth interferometric near-field spectroscopy by experimentally investigating the optical modes supported by individual $\mathrm{Sb}_{2} \mathrm{~S}_{3}$ nanodots deposited on an $\mathrm{Sb}_{2} \mathrm{~S}_{3}$ thin film. The near-field measurements reveal mode profiles reminiscent of low-order optical modes typical for more idealized cylindrical waveguides. These modes are seen in near-field spectra across the entire bandwidth supported by our laser while we find no clear signatures for spectrally sharp Fabry-Perot resonances of the nanodots. These results show that the $\mathrm{Sb}_{2} \mathrm{~S}_{3}$ nanodots can act as highrefractive-index dielectric waveguides with low losses in a broad spectral range below the bandgap. Together with their high 

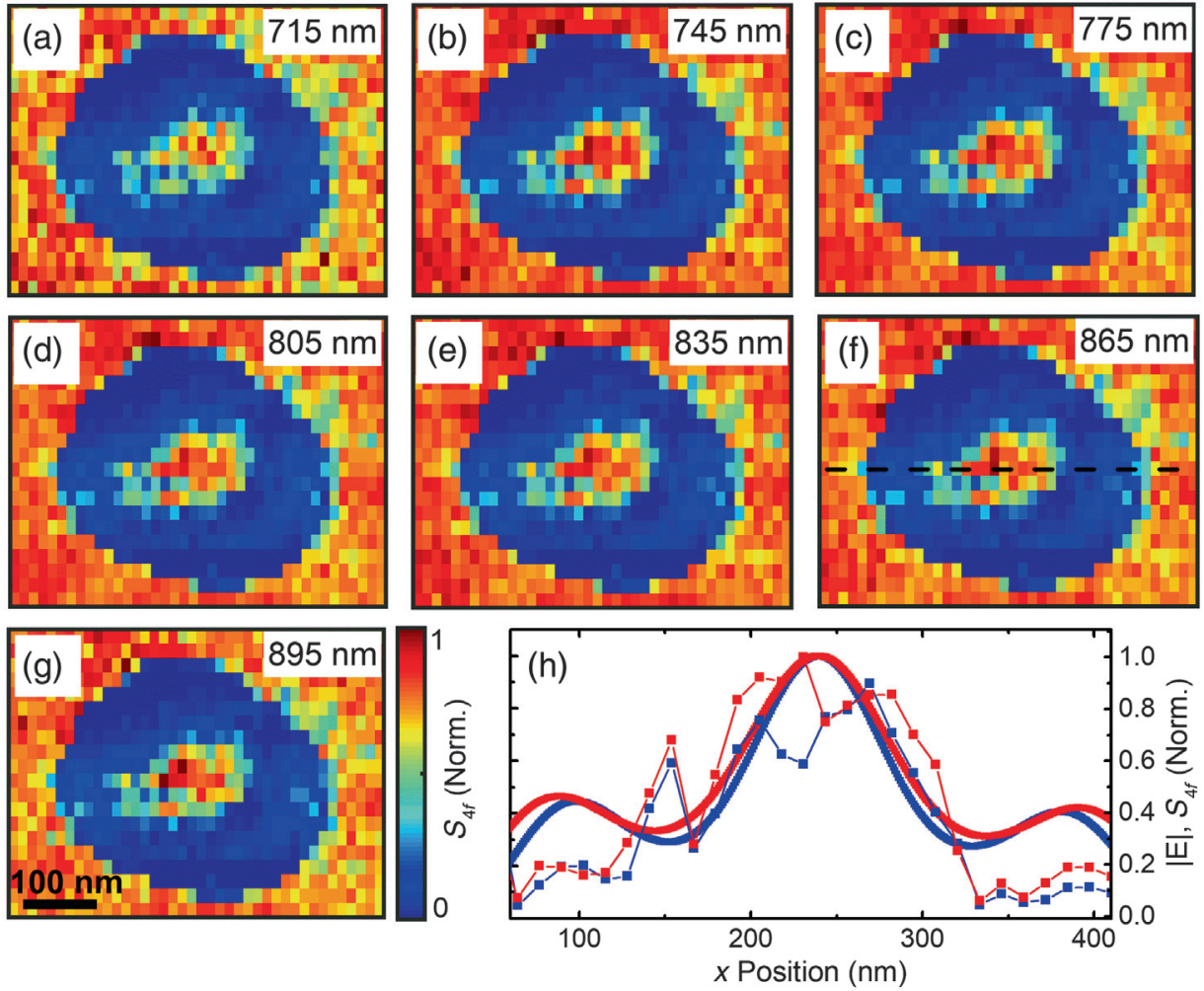

Fig. 7 Spectrally resolved near-field maps of an $\mathrm{Sb}_{2} \mathrm{~S}_{3}$ nanodot. (a)-(g) Near-field maps recreated from $30-\mathrm{nm}$ spectral bands centered at (a) $715 \mathrm{~nm}$, (b) $745 \mathrm{~nm}$, (c) $775 \mathrm{~nm}$, (d) $805 \mathrm{~nm}$, (e) $835 \mathrm{~nm}$, (f) $865 \mathrm{~nm}$, and (g) $895 \mathrm{~nm}$. (h) Cross cuts through the near-field maps shown in (a) (spectral range 700 to $730 \mathrm{~nm}$, blue symbols) and in (f) (spectral range 850 to $880 \mathrm{~nm}$, red symbols) together with the calculated SNOM maps at $715 \mathrm{~nm}$ (blue curve) and $865 \mathrm{~nm}$ (red curve).

above-bandgap absorption, their high photoconductivity, and the possibility for easy $2 \mathrm{D}$ and $3 \mathrm{D}$ structuring, this makes them promising candidates as switchable metamaterials. It will be highly interesting to investigate their nonlinear properties in future works.

\section{Acknowledgments}

We acknowledge funding by the Deutsche Forschungsgemeinschaft (SPP1391, SPP1839, GRK1885), the Niedersächsisches Ministerium für Wissenschaft und Kultur (LGRK, NanoEnergieforschung), the Korea Foundation for International Cooperation of Science and Technology (K20815000003), and the German-Israeli Foundation (1256). W. W. acknowledges financial support by the China Scholarship Council (CSC 201404910464).

\section{References}

1. R. Kondrotas, C. Chen, and J. Tang, " $\mathrm{Sb}_{2} \mathrm{~S}_{3}$ solar cells," Joule 2(5), 857-878 (2018).

2. M. Y. Versavel and J. A. Haber, "Structural and optical properties of amorphous and crystalline antimony sulfide thin-films," Thin Solid Films 515(18), 7171-7176 (2007).

3. P. P. Boix et al., "Hole transport and recombination in all-solid $\mathrm{Sb}_{2} \mathrm{~S}_{3}$-sensitized $\mathrm{TiO}_{2}$ solar cells using $\mathrm{CuSCN}$ as hole transporter," J. Phys. Chem. C 116(1), 1579-1587 (2012).

4. Y. C. Choi et al., "Highly improved $\mathrm{Sb}_{2} \mathrm{~S}_{3}$ sensitized-inorganicorganic heterojunction solar cells and quantification of traps by deep-level transient spectroscopy," Adv. Funct. Mater. 24(23), 3587-3592 (2014).
5. S. Messina, M. Nair, and P. Nair, "Antimony sulfide thin films in chemically deposited thin film photovoltaic cells," Thin Solid Films 515(15), 5777-5782 (2007).

6. J. A. Chang et al., "High-performance nanostructured inorganicorganic heterojunction solar cells," Nano Lett. 10(7), 2609-2612 (2010).

7. Y. C. Choi and S. I. Seok, "Efficient $\mathrm{Sb}_{2} \mathrm{~S}_{3}$-sensitized solar cells via single-step deposition of $\mathrm{Sb}_{2} \mathrm{~S}_{3}$ using $\mathrm{S} / \mathrm{Sb}$-ratio-controlled $\mathrm{SbCl}_{3}$-thiourea complex solution," Adv. Funct. Mater. 25(19), 2892-2898 (2015).

8. D.-H. Kim et al., "Highly reproducible planar $\mathrm{Sb}_{2} \mathrm{~S}_{3}$-sensitized solar cells based on atomic layer deposition," Nanoscale 6(23), 14549-14554 (2014).

9. W. Wang, P. Pfeiffer, and L. Schmidt-Mende, "Direct patterning of metal chalcogenide semiconductor materials," Adv. Funct. Mater. 30, 2002685 (2020).

10. M. Abulikemu et al., "Colloidal $\mathrm{Sb}_{2} \mathrm{~S}_{3}$ nanocrystals: synthesis, characterization and fabrication of solid-state semiconductor sensitized solar cells," J. Mater. Chem. A 4(18), 6809-6814 (2016).

11. W. Wang et al., "Hybrid solar cells from $\mathrm{Sb}_{2} \mathrm{~S}_{3}$ nanoparticle ink," Sol. Energ. Mat. Sol. C 172, 335-340 (2017).

12. M. R. Shcherbakov et al., "Ultrafast all-optical tuning of direct-gap semiconductor metasurfaces," Nat. Commun. 8, 17 (2017).

13. L. Novotny and B. Hecht, Principles of Nano-Optics, Cambridge University Press, Cambridge (2012).

14. T. Taubner, R. Hillenbrand, and F. Keilmann, "Performance of visible and mid-infrared scattering-type near-field optical microscopes," J. Microsc. 210(3), 311-314 (2003).

15. F. Zenhausern, Y. Martin, and H. Wickramasinghe, "Scanning interferometric apertureless microscopy: optical imaging at 10 angstrom resolution," Science 269(5227), 1083-1085 (1995). 
16. M. Balistreri et al., "Tracking femtosecond laser pulses in space and time," Science 294(5544), 1080-1082 (2001).

17. S. Götzinger et al., "Mapping and manipulating whispering gallery modes of a microsphere resonator with a near-field probe," J. Microsc. 202(1), 117-121 (2001).

18. F. Intonti et al., "Spectral tuning and near-field imaging of photonic crystal microcavities," Phys. Rev. B 78(4), 041401 (2008).

19. R. Esteban et al., "Direct near-field optical imaging of higher order plasmonic resonances," Nano Lett. 8(10), 3155-3159 (2008).

20. K. Imaeda, S. Hasegawa, and K. Imura, "Imaging of plasmonic eigen modes in gold triangular mesoplates by near-field optical microscopy," J. Phys. Chem. C 122(13), 7399-7409 (2018).

21. K. Imura, T. Nagahara, and H. Okamoto, "Imaging of surface plasmon and ultrafast dynamics in gold nanorods by near-field microscopy," J. Phys. Chem. B 108(42), 16344-16347 (2004).

22. R. L. Olmon et al., "Near-field imaging of optical antenna modes in the mid-infrared," Opt. Express 16(25), 20295-20305 (2008).

23. M. Rang et al., "Optical near-field mapping of plasmonic nanoprisms," Nano Lett. 8(10), 3357-3363 (2008).

24. A. Y. Frolov et al., "Near-field mapping of optical Fabry-Perot modes in all-dielectric nanoantennas," Nano Lett. 17(12), 76297637 (2017).

25. T. G. Habteyes et al., "Near-field mapping of optical modes on all-dielectric silicon nanodisks," ACS Photonics 2(9), 794-798 (2014).

26. R. Carminati et al., "Electromagnetic density of states in complex plasmonic systems," Surf. Sci. Rep. 70(1), 1-41 (2015).

27. G. C. des Francs et al., "Relationship between scanning near-field optical images and local density of photonic states," Chem. Phys. Lett. 345(5-6), 512-516 (2001).

28. K. Joulain et al., "Definition and measurement of the local density of electromagnetic states close to an interface," Phys. Rev. B 68(24), 245405 (2003).

29. V. Krachmalnicoff et al., "Towards a full characterization of a plasmonic nanostructure with a fluorescent near-field probe," Opt. Express 21(9), 11536-11545 (2013).

30. C. Chicanne et al., "Imaging the local density of states of optical corrals," Phys. Rev. Lett. 88(9), 097402 (2002).

31. M. Esmann et al., "Plasmonic nanofocusing spectral interferometry," Nanophotonics 9(2), 491-508 (2020).

32. E. Bailo and V. Deckert, "Tip-enhanced Raman spectroscopy of single RNA strands: towards a novel direct-sequencing method," Angew. Chem. Int. Ed. 47(9), 1658-1661 (2008).

33. S. F. Becker et al., "Gap-plasmon-enhanced nanofocusing nearfield microscopy," ACS Photonics 3(2), 223-232 (2016).

34. R. Zhang et al., "Chemical mapping of a single molecule by plasmon-enhanced Raman scattering," Nature 498, 82-86 (2013).

35. M. Esmann et al., "Vectorial near-field coupling," Nat. Nanotechnol. 14(7), 698-704 (2019)

36. C. C. Neacsu et al., "Near-field localization in plasmonic superfocusing: a nanoemitter on a tip," Nano Lett. 10(2), 592-596 (2010).

37. C. Ropers et al., "Grating-coupling of surface plasmons onto metallic tips: a nanoconfined light source," Nano Lett. 7(9), 27842788 (2007).

38. E. Ash and G. Nicholls, "Super-resolution aperture scanning microscope," Nature 237, 510-512 (1972).

39. R. Hillenbrand, B. Knoll, and F. Keilmann, "Pure optical contrast in scattering-type scanning near-field microscopy," J. Microsc. 202(1), 77-83 (2001).

40. B. Knoll and F. Keilmann, "Enhanced dielectric contrast in scattering-type scanning near-field optical microscopy," Opt. Commun. 182(4-6), 321-328 (2000).

41. N. Ocelic, A. Huber, and R. Hillenbrand, "Pseudoheterodyne detection for background-free near-field spectroscopy," Appl. Phys. Lett. 89(10), 101124 (2006).

42. Y. Sasaki and H. Sasaki, "Heterodyne detection for the extraction of the probe-scattering signal in scattering-type scanning near- field optical microscope," Jpn. J. Appl. Phys. 39, L321-L323 (2000).

43. R. Hillenbrand and F. Keilmann, "Complex optical constants on a subwavelength scale," Phys. Rev. Lett. 85(14), 3029-3032 (2000).

44. J. Brauer et al., "In-line interferometer for broadband near-field scanning optical spectroscopy," Opt. Express 25(13), 1550415525 (2017).

45. D. Gloge, "Weakly guiding fibers," Appl. Opt. 10(10), 2252-2258 (1971).

46. F. Mitschke, Fiber Optics, Springer-Verlag, Berlin (2016).

47. X. Wang et al., "A fast chemical approach towards $\mathrm{Sb}_{2} \mathrm{~S}_{3}$ film with a large grain size for high-performance planar heterojunction solar cells," Nanoscale 9(10), 3386-3390 (2017).

48. S. Schmidt et al., "Adiabatic nanofocusing on ultrasmooth singlecrystalline gold tapers creates a 10-nm-sized light source with few-cycle time resolution," ACS Nano 6(7), 6040-6048 (2012).

49. R. L. Olmon et al., "Optical dielectric function of gold," Phys. Rev. $B$ 86(23), 235147 (2012).

50. P. Groß et al., "Plasmonic nanofocusing-grey holes for light," Adv. Phys. X 2(2), 297-330 (2016).

51. N. Talebi et al., "Excitation of mesoscopic plasmonic tapers by relativistic electrons: phase matching versus eigenmode resonances," ACS Nano 9(7), 7641-7648 (2015).

52. B. Piglosiewicz et al., "Carrier-envelope phase effects on the strong-field photoemission of electrons from metallic nanostructures," Nat. Photonics 8(1), 37-42 (2014).

53. Lumerical FDTD Solutions, www.lumerical.com (accessed 9 September 2019).

54. S. Grésillon et al., "Experimental observation of localized optical excitations in random metal-dielectric films," Phys. Rev. Lett. 82(22), 4520-4523 (1999).

55. J. Zhong et al., "Strong spatial and spectral localization of surface plasmons in individual randomly disordered gold nanosponges," Nano Lett. 18(8), 4957-4964 (2018).

Jinxin Zhan is a PhD student in the Near-Field Photonics Laboratory of Professor Christoph Lienau at University of Oldenburg. Her current interests are focused on broadband interferometric homodyne scanning near-field spectroscopy to study optical resonances of individual nanostructures and coupled plasmonic systems. She graduated in June 2014 at the Shanghai Institute of Optics \& Fine Mechanics, Chinese Academy of Sciences.

Wei Wang received his PhD from the Physics Department of Konstanz University in 2019. His works have been focused on fabrication of semiconductor nanostructures, such as nanoparticle, 2D/3D periodic patterns for optoelectronic and photovoltaic applications.

Jens Brauer works as a software developer for Bertrandt and BMW in Munich, Germany. He graduated from University of Oldenburg in 2013 and will defend his PhD thesis in 2020. His research interests are nano optics and near-field imaging

Lukas Schmidt-Mende: Biography is not available.

Christoph Lienau is a professor in experimental physics at the University of Oldenburg. After receiving his $\mathrm{PhD}$ in physical chemistry in Göttingen, he worked with Ahmed $\mathrm{H}$. Zewail at Caltech and then as a scientific staff member at Max Born Institute. In 2006, he became a full professor in physics. He has authored more than 200 publications and is a fellow of OSA. His research interests are in ultrafast, nano, and quantum optics.

Petra Groß is a researcher at the Institute for Physics at University of Oldenburg. She received her PhD from University of Twente in 2003 and her postdoctoral lecture qualification from University of Münster in 2014. Her research interests are in imaging, ultrafast, nonlinear and nano optics, and ultrafast electron microscopy. 